\title{
Ratification vote on taxonomic proposals to the International Committee on Taxonomy of Viruses (2008)
}

\author{
E. B. Carstens $\cdot$ L. A. Ball
}

Received: 26 April 2009/Accepted: 12 May 2009/Published online: 3 June 2009

(C) Springer-Verlag 2009

\begin{abstract}
In accordance with the Statutes of the International Committee of Taxonomy of Viruses (ICTV), the final stage in the process of making changes to the Universal Scheme of Virus Classification is the ratification of taxonomic proposals by ICTV Members. This can occur either at a Plenary meeting of ICTV, held during an International Congress of Virology meeting, or by circulation of proposals by mail followed by a ballot. Therefore, a list of proposals that had been subjected to the full, multistage review process was prepared and presented on the ICTVonline web pages in March 2008. This review process involved input from the ICTV Study Groups and Subcommittees, other interested virologists, and the ICTV Executive Committee. For the first time, the ratification process was performed entirely by email. The proposals were sent electronically via email on 18 March 2008 to ICTV Life Members (11), ICTV Subcommittee Members (74), and ICTV National Representatives (53).
\end{abstract}

\section{Introduction}

In accordance with the Statutes of the International Committee on Taxonomy of Viruses (ICTV), the final stage in

E. B. Carstens $(\bowtie)$

Department of Microbiology and Immunology,

Queen's University, Kingston, ON K7L 3N6, Canada

e-mail: carstens@queensu.ca

L. A. Ball

Department of Microbiology, University of Alabama

at Birmingham, Birmingham, AL 35294, USA

e-mail: andyb@uab.edu the process of making changes to the Universal Scheme of Virus Classification is the ratification of taxonomic proposals by ICTV members. This can occur either at a plenary meeting of ICTV, held during an International Congress of Virology meeting, or by circulation of proposals by mail, followed by a ballot. Therefore, a list of proposals that had been subjected to the full, multi-stage review process was prepared and presented on the ICTVonline web pages in March 2008. This review process involved input from the ICTV study groups and subcommittees, other interested virologists, and the ICTV Executive Committee. For the first time, the ratification process was performed entirely by email. The proposals were sent electronically via email on 18 March 2008, to ICTV Life Members (11), ICTV Subcommittee Members (74), and ICTV National Representatives (53). Members were then requested to vote on whether to ratify the taxonomic proposals, within a 1-month deadline.

International Committee on Taxonomy of Viruses attempts to keep an up-to-date list of all members, including National Representatives, but this is a daunting task. As a means of promoting contact between ICTV and its National Members and to ensure that lists of members are up to date, member societies of IUMS are obliged to either renew ICTV National Members or replace them every three years. In effect, National Members have a 3 -year tenure that can be renewed indefinitely. National Societies, which are members of the International Union of Microbiological Societies, are encouraged to contact ICTV directly through the ICTV Secretary with the names of their National Representatives. Complete contact information can be obtained from the ICTV web site: http://ictvonline.org.

The following are the taxonomic proposals that were ratified by ICTV members in April 2008. 


\section{Vertebrate Virus Subcommittee}

Adenoviruses

2005.223V.04 To create a species in the genus Atadenovirus, named Snake adenovirus A.

2005.224V.04 To create a species in the genus Mastadenovirus, named Simian adenovirus A.

Birnaviruses

2005.212V-215V.04 To create a new genus, Blosnavirus, in the family Birnaviridae with Blotched snakehead virus as the type species.

2005.216V-222V.04 To create a new family, Picobirnaviridae, to contain a new genus Picobirnavirus, with Human picobirnavirus as the type species, and Rabbit picobirnavirus as a designated species.

Bunyaviruses

2005.119V.04 To create a species in the genus Hantavirus in the family Bunyaviridae, named Saaremaa virus.

Circoviruses

2004.007V.04 To create a species in the genus Circovirus in the family Circoviridae, named Duck circovirus.

2007.081V.04 To create the following species in the genus Circovirus, in the family Circoviridae named Finch circovirus, Gull circovirus, Starling circovirus.

Coronaviruses

2005.260V.04 To create the following species in the genus Coronavirus in the family Coronaviridae, named Goose coronavirus, Pigeon coronavirus, Duck coronavirus.

2006.009V.04 To create a species in the genus Coronavirus in the family Coronaviridae, named Human coronavirus NL63.

2006.010V.04 To create a species in the genus Coronavirus in the family Coronaviridae, named Human coronavirus HKU1.

2006.011V.04 To create a species in the genus Coronavirus in the family Coronaviridae, named Equine coronavirus.

Herpesviruses

Summary

2005.020-72V.04 This large set of proposals from the Herpesviridae Study Group achieves a far-reaching update of herpesvirus taxonomy. It includes:
Establishment of the order Herpesvirales.

Assignment of the Herpesviridae and two new families to the order.

Transfer of genus Ictalurivirus to the new family Alloherpesviridae.

Population of the Alloherpesviridae with fish and amphibian viruses.

Establishment of a genus, Ostreavirus, in the new family Malacoherpesviridae.

Assignment of Ostreid herpesvirus 1 to the genus Ostreavirus.

Creation of the new genera Macavirus and Percavirus in subfamily Gammaherpesvirinae.

Creation of a new genus, Proboscivirus, in subfamily Betaherpesvirinae.

Assignments of species to various genera in the family Herpesviridae.

Changing names of old world non-human primate viruses to refer to host genus.

\section{Details}

2005.020V.04 To remove the following species from the genus Rhadinovirus of the subfamily.

Gammaherpesvirinae in the family Herpesviridae: Alcelaphine herpesvirus 1, Alcelaphine herpesvirus 2, Equid herpesvirus 2, Equid herpesvirus 5, Equid herpesvirus 7, Hippotragine herpesvirus 1, Mustelid herpesvirus 1, Ovine herpesvirus 2. (These species are reassigned to new taxa below).

2005.021V.04 To remove the following unassigned viruses from the family Herpesviridae:

Acipenserid herpesvirus 1, Acipenserid herpesvirus 2, Anguillid herpesvirus 1, Cyprinid herpesvirus 1, Cyprinid herpesvirus 2, Esocid herpesvirus 1, Percid herpesvirus 1, Pleuronectid herpesvirus 1, Ranid herpesvirus 1, Ranid herpesvirus 2, Salmonid herpesvirus 1, Salmonid herpesvirus 2, Ostreid herpesvirus 1, Ateline herpesvirus 3, Columbid herpesvirus 1, Elephantid herpesvirus 1, Suid herpesvirus 2 .

(This is not strictly necessary as a taxonomic proposal because it concerns entities below the species level, but it is left in to clarify this reorganization of the Herpesviridae. These viruses are reassigned to new taxa below).

\section{Order Herpesvirales}

2005.022-023V.04 To create a new order in the dsDNA viruses, named Herpesvirales.

2005.024V.04 To designate the following family in the order Herpesvirales: Herpesviridae. 
Family Alloherpesviridae

2005.025-026V.04 To create a new family in the order Herpesvirales, named Alloherpesviridae.

2005.027V.04 To designate the following genus in the family Alloherpesviridae:Ictalurivirus.

2005.028V.04 To designate the following unassigned viruses in the family Alloherpesviridae:

Acipenserid herpesvirus 1, Acipenserid herpesvirus 2, Anguillid herpesvirus 1, Cyprinid herpesvirus 1, Cyprinid herpesvirus 2, Esocid herpesvirus 1, Percid herpesvirus 1, Pleuronectid herpesvirus 1, Ranid herpesvirus 1, Ranid herpesvirus 2, Salmonid herpesvirus 1, Salmonid herpesvirus 2. (This is not strictly necessary as a taxonomic proposal because it concerns entities below the species level, but it is left in to clarify this reorganization of the Herpesviridae).

2005.029V.04 To create the following unassigned species in the family Alloherpesviridae:

Cyprinid herpesvirus 3.

\section{Family Malacoherpesviridae}

2005.030-031V.04 To create a new family in the order Herpesvirales, named Malacoherpesviridae.

2005.032-033V.04 To create a new genus in the family Malacoherpesviridae, named Ostreavirus.

2005.034-035V.04 To designate Ostreid herpesvirus 1 the type species of the genus Ostreavirus.

\section{Family Herpesviridae}

2005.036-037V.04 To create a new genus in the subfamily Gammaherpesvirinae in the family Herpesviridae, named Macavirus.

2005.038V.04 To designate Alcelaphine herpesvirus 1 as the type species of the genus Macavirus.

2005.039V.04 To designate the following species in the genus Macavirus: Alcelaphine herpesvirus 1, Alcelaphine herpesvirus 2, Hippotragine herpesvirus 1, Ovine herpesvirus 2.

2005.040V.04 To create the following species in the genus Macavirus: Bovine herpesvirus 6, Caprine herpesvirus 2 .

2005.041V.04 To create the following species in the genus Macavirus: Suid herpesvirus 3.

2005.042V.04 To create the following species in the genus Macavirus: Suid herpesvirus 4.

2005.043V.04 To create the following species in the genus Macavirus: Suid herpesvirus 5.

2005.044-046V.04 To create a new genus in the subfamily Gammaherpesvirinae in the family Herpesviridae, named Percavirus, and to designate the species Equid herpesvirus 2 as the type species.

2005.047V.04 To designate the following species in the genus Percavirus: Equid herpesvirus 2, Equid herpesvirus 5, Mustelid herpesvirus 1.

2005.048V.04 To create the following unassigned species in the subfamily Gammaherpesvirinae: Equid herpesvirus 7.

2005.049-050V.04 To create a new genus in the subfamily Betaherpesvirinae in the family Herpesviridae, named Proboscivirus.

2005.051-052V.04 To designate the species Elephantid herpesvirus 1 as the type species of the genus Proboscivirus.

2005.053V.04 To create the following species in the genus Mardivirus in the subfamily Alphaherpesvirinae in the family Herpesviridae: Columbid herpesvirus 1 .

2005.054V.04 To designate the following species in the genus Iltovirus in the subfamily Alphaherpesvirinae in the family Herpesviridae: Psittacid herpesvirus 1.

2005.055V.04 To create the following species in the genus Rhadinovirus in the subfamily Gammaherpesvirinae in the family Herpesviridae: Ateline herpesvirus 3.

2005.056V.04 To create the following unassigned species in the subfamily Betaherpesvirinae in the family Herpesviridae: Suid herpesvirus 2.

2005.057-058V.04 To create the following unassigned species in the subfamily Alphaherpesvirinae in the family Herpesviridae: Chelonid herpesvirus 5, Chelonid herpesvirus 6.

2005.059V.04 To create the following unassigned species in the subfamily Gammaherpesvirinae in the family Herpesviridae: Phocid herpesvirus 2.

2005.060V.04 To create the following unassigned species in the family Herpesviridae: Iguanid herpesvirus 2.

\section{Changes to species names and virus names}

2005.061V.04 To rename Cercopithecine herpesvirus 1 in the genus Simplexvirus in the family Herpesviridae as Macacine herpesvirus 1.

2005.062V.04 To rename Cercopithecine herpesvirus 16 in the genus Simplexvirus in the family Herpesviridae as Papiine herpesvirus 2.

2005.063V.04 To rename Cercopithecine herpesvirus 8 in the genus Cytomegalovirus in the family Herpesviridae as Macacine herpesvirus 3.

2005.064V.04 To rename Pongine herpesvirus 4 in the genus Cytomegalovirus in the family Herpesviridae as Panine herpesvirus 2.

2005.065V.04 To rename Cercopithecine herpesvirus 12 in the genus Lymphocryptovirus in the family Herpesviridae as Papiine herpesvirus 1. 
2005.066V.04 To rename Cercopithecine herpesvirus 15 in the genus Lymphocryptovirus in the family Herpesviridae as Macacine herpesvirus 4.

2005.067V.04 To rename Pongine herpesvirus 1 in the genus Lymphocryptovirus in the family Herpesviridae as Panine herpesvirus 1.

2005.068V.04 To rename Pongine herpesvirus 3 in the genus Lymphocryptovirus in the family Herpesviridae as Gorilline herpesvirus 1.

2005.069V.04 To rename Cercopithecine herpesvirus 17 in the genus Rhadinovirus in the family Herpesviridae as Macacine herpesvirus 5.

2005.070V.04 To rename Cercopithecine herpesvirus 10, an unassigned virus in the family Herpesviridae: Macacine herpesvirus 6.

2005.071V.04 To rename Cercopithecine herpesvirus 13 an unassigned virus in the family Herpesviridae: Macacine herpesvirus 7.

(2005.070-071V are not strictly necessary as taxonomic proposals because they concern entities below the species level, but they are left in to clarify this reorganization of the Herpesviridae).

2005.072V.04 To rename Callitrichine herpesvirus 1 , an unassigned species in the subfamily Gammaherpesvirinae in the family Herpesviridae: Saguinine herpesvirus 1.

\section{Picornaviruses}

2005.261V.04 To remove the following species from the existing genus Enterovirus in the family Picornaviridae: Poliovirus. (Note: Poliovirus hereby loses its status as a virus species).

2005.262V.04 To assign the following viruses to the species Human enterovirus $C$ in the existing genus Enterovirus in the family Picornaviridae: Human poliovirus 1, Human poliovirus 2, Human poliovirus 3 .

(This is not strictly necessary as a taxonomic proposal because it concerns entities below the species level, but it is left in to clarify this reorganization of the Picornaviridae).

2005.263V.04 To change the type species of the genus Enterovirus in the family Picornaviridae, from Poliovirus to Human enterovirus $C$.

2005.264V.04 To remove the following species from the existing genus Rhinovirus in the family Picornaviridae: Human rhinovirus A, Human rhinovirus $B$.

2005.265V.04 To assign the following species to the genus Enterovirus in the family Picornaviridae: Human rhinovirus A, Human rhinovirus $B$.

2005.266V.04 To remove the existing genus Rhinovirus from the family Picornaviridae. (Note: The genus Rhinovirus hereby disappears).
2005.200G.04-201G.04 To create an order to accommodate picornavirus-like viruses and to name the order Picornavirales.

2005.202G.04 To designate the following families and genera as members of the order created in 2005.200G, named Picornaviridae, Dicistroviridae, Marnaviridae, Iflavirus, Sequiviridae, Sadwavirus, Cheravirus, Comoviridae.

\section{Poxviruses}

2006.022-023V.04 To remove Squirrel parapoxvirus from the genus Parapoxvirus in the family Poxviridae and rename it Squirrel poxvirus as an unssigned species in the subfamily Chordopoxvirinae in the family Poxviridae.

2006.024-026V.04 To create the new genus Cervidpoxvirus in the subfamily Chordopoxvirinae in the family Poxviridae with Deerpox virus $W-848-83$ as the type species.

Reoviruses

2005.225V-228V.04 To create the new genus Mimoreovirus within the family Reoviridae with Micromonas pusilla reovirus as the type species of the new genus.

2005.229V-232V.04 To create the new genus Dinovernavirus within the family Reoviridae with Aedes pseudoscutellaris reovirus as the type species of the new genus.

2005.242V-246V.04 To create the new genus Cardoreovirus within the family Reoviridae with Eriocheir sinensis reovirus as the type species of the new genus.

2007.137V.04 To create a species in the genus Orbivirus in the family Reoviridae, named Yunnan orbivirus.

2007.138V.04 To create a species in the genus Aquareovirus in the family Reoviridae, named Aquareovirus $G$.

\section{Invertebrate Virus Subcommittee}

Baculoviruses

2006.033I.04 To rename the existing genus Granulovirus in the family Baculoviridae: Betabaculovirus.

2006.034I.04 To remove from the family Baculoviridae the genus Nucleopolyhedrovirus and replace it with three genera.

2006.035I-036I.04 To create a new genus in the family Baculoviridae and to name it Alphabaculovirus.

2006.037I.04 To designate the following as the type species in the new genus created in 2006.035I: Autographa californica multiple nucleopolyhedrovirus.

2006.038I.04 To assign the following species in the new genus created in 2006.035I: Adoxophyes honmai nucleopolyhedrovirus, Agrotis ipsilon nucleopolyhedrovirus, 
Anticarsia gemmatalis multiple nucleopolyhedrovirus, Autographa californica multiple nucleopolyhedrovirus, Bombyx mori nucleopolyhedrovirus, Buzura suppressaria nucleopolyhedrovirus, Choristoneura fumiferana DEF multiple nucleopolyhedrovirus, Choristoneura fumiferana multiple nucleopolyhedrovirus, Choristoneura rosaceana nucleopolyhedrovirus, Ectropis obliqua nucleopolyhedrovirus, Epiphyas postvittana nucleopolyhedrovirus, Helicoverpa armigera nucleopolyhedrovirus, Helicoverpa zea single nucleopolyhedrovirus, Lymantria dispar multiple nucleopolyhedrovirus, Mamestra brassicae multiple nucleopolyhedrovirus, Mamestra configurata nucleopolyhedrovirus A, Mamestra configurata nucleopolyhedrovirus $B$, Orgyia pseudotsugata multiple nucleopolyhedrovirus, Spodoptera exigua multiple nucleopolyhedrovirus, Spodoptera frugiperda multiple nucleopolyhedrovirus, Spodoptera litura nucleopolyhedrovirus, Thysanoplusia orichalcea nucleopolyhedrovirus, Trichoplusia ni single nucleopolyhedrovirus, Wiseana signata nucleopolyhedrovirus.

2006.040I-041I.04 To create a new genus in the family Baculoviridae and to name it Gammabaculovirus.

2006.042I.04 To designate as the type species in the new genus created in 2006.040I Neodiprion lecontei nucleopolyhedrovirus.

2006.043I.04 To assign the following species in the new genus created in 2006.040I: Neodiprion lecontei nucleopolyhedrovirus, Neodiprion sertifer nucleopolyhedrovirus.

2006.044I-045I.04 To create a new genus in the family Baculoviridae and to name it Deltabaculovirus.

2006.046I.04 To create the species Culex nigripalpus nucleopolyhedrovirus and designate it as the type species in the new genus created in 2006.044I.

2006.047I.04 To assign the following species in the new genus created in 2006.044I: Culex nigripalpus nucleopolyhedrovirus.

\section{Dicistroviruses}

2005.118I.04 To create the following unassigned species in the family Dicistroviridae: Kashmir bee virus, Solenopsis invicta virus-1.

Iflaviruses

2005.120I.04 To create the following species in the genus Iflavirus: Deformed wing virus, Ectropis obliqua virus, Varroa destructor virus-1.

2006.029I-030I.04 To create a new family to host the genus Iflavirus and to name it Iflaviridae.

2006.031I.04 To assign the genus Iflavirus to the new family created in 2006.030I.04
Nimaviruses

2007.084I.04 To rename the species White spot syndrome virus 1 in the genus Whispovirus in the family Nimaviridae White spot syndrome virus.

Tetraviruses

2005.259I.04 To create a species in the genus Omegatetravirus in the family Tetraviridae named Dendrolimus punctatus virus.

\section{Plant Virus Subcommittee}

Bromoviruses

2004.008P-009P.04 To create a new genus of plant viruses with quasi-spherical particles $25-35 \mathrm{~nm}$ in diameter and a tripartite ssRNA genome in the family Bromoviridae and to name it Anulavirus.

2004.010P.04 To designate Pelargonium zonate spot virus as the type species of the genus Anulavirus.

2004.011P.04 To create a species in the genus Anulavirus, named: Pelargonium zonate spot virus.

\section{Carmoviruses}

2007.121P.04 To create two species in the genus Carmovirus in the family Tombusviridae, named Angelonia flower break virus, Pea stem necrosis virus.

Cheraviruses

2007.064P.04 To create a new species in the unassigned genus Cheravirus, named Stocky prune virus.

\section{Closteroviruses}

2006.002P.04 To create a species in the genus Closterovirus in the family Closteroviridae, named Mint virus 1.

2006.004P.04 To create an unassigned species in the family Closteroviridae, named Mint vein banding-associated virus.

2006.006P.04 To create a species in the genus Crinivirus in the family Closteroviridae, named Strawberry pallidosis-associated virus.

2007.004.04-005P.04 To create two species in the genus Crinivirus in the family Closteroviridae, named Potato yellow vein virus, Blackberry yellow vein-associated virus. 


\section{Comoviruses}

2005.007P.04 To create two new species in the genus Nepovirus in the family Comoviridae named Grapevine deformation virus, Grapevine Anatolian ringspot virus.

2007.065P.04 To create a new species in the genus Fabavirus in the family Comoviridae, named Gentian mosaic virus.

\section{Endornaviruses}

2006.019P.04-020P.04 To create a new family to host the unassigned genus Endornavirus and to name it Endornaviridae.

Flexiviruses

2005.006P.04 To change the name of Rupestris stem pitting-associated virus to Grapevine rupestris stem pittingassociated virus, in the genus Foveavirus in the family Flexiviridae.

2005.008P.04 To create a new species in the genus Trichovirus in the family Flexiviridae, named Apricot pseudo-chlorotic leaf spot virus.

2005.009P.04 To create two new species in the genus Carlavirus in the family Flexiviridae, named: Daphne virus $S$, Melon yellowing-associated virus.

2007.030P.04 To create three species in the genus Carlavirus, named Narcissus symptomless virus, Potato virus $P$, Sweet potato chlorotic fleck virus.

2005.010P.04 To create three new species in the genus Potexvirus in the family Flexiviridae, named Opuntia virus $X$, Schlumbergera virus $X$, Zygocactus virus $X$.

2005.017P-018P.04 To create a new genus in the family Flexiviridae and to name it Citrivirus.

2005.019P.04 To name Citrus leaf blotch virus as type species of the new genus in the family Flexiviridae created in 2005.017P.

2006.003P.04 To remove the following from the list of approved species in the genus Capillovirus in the family Flexiviridae: Lilac chlorotic leafspot virus.

2006.007P.04 To create two species in the genus Potexvirus in the family Flexiviridae, named Alstroemeria virus $X$, Mint virus $X$.

2006.012P.04 To remove a species from the genus Potexvirus in the family Flexiviridae, named Scallion virus $X$.

Geminiviruses

2005.001P.04 To create a new species in the genus Curtovirus in the family Geminiviridae, named Spinach curly top virus.
2007.003P.04 To create a species in the genus Curtovirus in the family Geminiviridae, named Pepper curly top virus.

2005.002P.04 To create 24 new species in the genus Begomovirus in the family Geminiviridae, named Ageratum leaf curl virus, Corchorus yellow vein Vietnam virus, Eupatorium yellow vein mosaic virus, Honeysuckle yellow vein Kagoshima virus, Horsegram yellow mosaic virus, Lindernia anagallis yellow vein virus, Malvastrum yellow vein Yunnan virus, Okra yellow mosaic Mexico virus, Pepper yellow vein Mali virus, Senecio yellow mosaic virus, Sida micrantha mosaic virus, Sida yellow mosaic China virus, Tomato leaf curl Guangdong virus, Tomato leaf curl Java virus, Tomato leaf curl Joydebpur virus, Tomato leaf curl Madagascar virus, Tomato leaf curl Mali virus, Tomato leaf curl Mayotte virus, Tomato mild yellow leaf curl Aragua virus, Tomato mosaic leaf curl virus, Tomato yellow leaf curl Axarquia virus, Tomato yellow leaf curl Guangdong virus, Tomato yellow leaf curl Mali virus, Tomato yellow margin leaf curl virus.

2006.001P.04 To create eighteen new species in the genus Begomovirus in the family Geminiviridae, named Alternanthera yellow vein virus, Cabbage leaf curl Jamaica virus, Cotton leaf curl Bangalore virus, East African cassava mosaic Kenya virus, Ludwigia yellow vein virus, Malvastrum leaf curl Guangdong virus, Malvastrum leaf curl virus, Malvastrum yellow mosaic virus, Okra yellow mottle Iguala virus, Rhynchosia golden mosaic Sinaloa virus, Sida leaf curl virus, Siegesbeckia yellow vein virus, Siegesbeckia yellow vein Guangxi virus, Tobacco leaf curl Cuba virus, Tomato leaf curl Guangxi virus, Tomato leaf curl Uganda virus, Tomato yellow spot virus, Vernonia yellow vein virus.

2007.001P.04 To create 43 species in the genus Begomovirus in the family Geminiviridae, named Ageratum yellow vein Hualian virus, Bitter gourd yellow vein virus, Boerhavia yellow spot virus, Clerodendron golden mosaic virus, Corchorus golden mosaic virus, Corchorus yellow spot virus, Desmodium leaf distortion virus, Dicliptera yellow mottle Cuba virus, Erectites yellow mosaic virus, Euphorbia leaf curl Guangxi virus, Euphorbia mosaic virus, Kudzu mosaic virus, Ludwigia yellow vein Vietnam virus, Malvastrum yellow leaf curl virus, Mesta yellow vein mosaic virus, Mimosa yellow leaf curl virus, Okra yellow crinkle virus, Pedilenthus leaf curl virus, Pepper leaf curl Lahore virus, Pepper leaf curl Pakistan virus, Pepper yellow leaf curl Indonesia virus, Pumpkin yellow mosaic virus, Radish leaf curl virus, Sida yellow mosaic Yucatan virus, Sida yellow vein Madurai virus, Sida yellow vein Vietnam virus, Soybean blistering mosaic virus, Spilanthes yellow vein virus, Sweet potato leaf curl Canary virus, Sweet potato leaf curl China virus, Sweet potato leaf curl Lanzarote virus, Sweet potato leaf curl Spain virus, Tomato 
leaf curl Arusha virus, Tomato leaf curl Comoros virus, Tomato leaf curl Hsinchu virus, Tomato leaf curl Kerala virus, Tomato leaf curl Pakistan virus, Tomato leaf curl Pune virus, Tomato leaf curl Seychelles virus, Tomato leaf curl Sinaloa virus, Tomato yellow leaf curl Indonesia virus, Tomato yellow leaf curl Vietnam virus, Tomato yellow vein streak virus.

2007.002P.04 To remove the following six species from the genus Begomovirus in the family Geminiviridae:Ageratum yellow vein China virus, Ageratum yellow vein Taiwan virus, Cotton leaf curl Rajasthan virus, Potato yellow mosaic Trinidad virus, Tobacco leaf curl Kochi virus, Tomato leaf curl Iran virus.

Ipomoviruses

2007.072P.04 To create a species in the genus Ipomovirus, named Squash vein yellowing virus.

\section{Luteoviruses}

2005.014P.04 To create a new species in the genus Polerovirus in the family Luteoviridae, named Carrot red leaf virus.

2005.016P.04 To remove an unassigned species from the family Luteoviridae, named Strawberry mild yellow edge-associated virus.

Macluraviruses

2007.071P.04 To create three species in the genus Macluravirus, named Alpinia mosaic virus, Chinese yam necrotic mosaic virus, Ranunculus latent virus.

Marafiviruses

2005.122P.04 To create a new species in the genus Marafivirus in the family Tymoviridae, named Citrus sudden death-associated virus.

Ophioviruses

2005.233-235P.04 To create a new family to host the unassigned genus Ophiovirus and to name it Ophioviridae.

Polemoviruses

2005.249-252P.04 To create a new unassigned genus named Polemovirus to accommodate the new species Poinsettia latent virus, formerly known as Poinsettia crytic virus, which was previously a tentative species in the genus Alphacryptovirus in the family Partitiviridae.
Potyviruses

2007.073P.04 To create twenty species in the genus Potyvirus, named.

Amazon lily mosaic virus, Basella rugose mosaic virus, Chinese artichoke mosaic virus, Daphne mosaic virus, East Asian Passiflora virus, Euphorbia ringspot virus, Fritillary virus $Y$, Meadow saffron breaking virus, Passiflora chlorosis virus, Pennisetum mosaic virus, Pfaffia mosaic virus, Ranunculus leaf distortion virus, Ranunculus mild mosaic virus, Ranunculus mosaic virus, Spiranthes mosaic virus 2, Sweet potato virus 2, Thunberg fritillary mosaic virus, Tradescantia mild mosaic virus, Tuberose mild mottle virus, Zantedeschia mild mosaic virus.

2007.074P.04 To remove the species Zantedeschia mosaic virus from the genus Potyvirus.

Tombusviruses

2005.011P.04 To create a new species in the genus $\mathrm{Nec}$ rovirus in the family Tombusviridae, named Olive mild mosaic virus.

2005.012P.04 To create a new species in the genus Tombusvirus in the family Tombusviridae, named Havel River virus.

2007.123P.04 To create two new species in the genus Tombusvirus in the family Tombusviridae, named Limonium flower distortion virus, Pelargonium necrotic spot virus.

2007.125P.04 To remove the species Pear latent virus from the genus Tombusvirus.

Tymoviruses

2007.063P.04 To create two species in the genus Tymovirus, named Anagyris vein yellowing virus, Nemesia ring necrosis virus.

Viroids

2005.253P.04 To change the name of a species in the genus Apscaviroid in the family Pospiviroidae: from Citrus viroid III to Citrus dwarfing viroid.

2005.254P.04 To change the name of a species in the genus Cocaviroid in the family Pospiviroidae: from Citrus viroid IV to Citrus bark cracking viroid.

2005.255P-256P.04 To create a new genus and to name it Elaviroid.

2005.257P.04 To assign the species Eggplant latent viroid as the type species of the new genus created in 2005.255P.

2005.258P.04 To assign the species Eggplant latent viroid as a species of the new genus created in 2005.255P. 


\section{Fungus Virus Subcommittee}

Mimiviruses

2005.004F.04-005F.04 To create a new family to accommodate the genus Mimivirus and to name it Mimiviridae.

\section{Prokaryote Virus Subcommittee}

Ampullaviruses

2005.084B-085B.04 To create a new genus and to name it Ampullavirus.

2005.086B.04 To designate the species Acidianus bottle-shaped virus as the type species of the new genus created in 2005.084B.

2005.087B.04 To create the following as a species of the new genus created in 2005.084B: Acidianus bottle-shaped virus.

2005.088B-089B.04 To create a new family and to name it Ampullaviridae.

2005.090B.04 To designate the following genus as part of the new family created in 2005.088B: Ampullavirus.

\section{Globuloviruses}

2005.077-078B.04 To create a new unassigned genus named Globulovirus.

2005.079B.04 To designate the species Pyrobaculum spherical virus as the type species of the new genus created in 2005.078B.

2005.080B.04 To create the following as a species of the new genus created in 2005.078B: Pyrobaculum spherical virus.

2005.081B-082B.04 To create a new family and to name it Globuloviridae.

2005.083B.04 To designate the following genera as belonging to the new family created in 2005.081B: Globulovirus.

2007.085B.04 To create the following as species in the genus Globulovirus in the family Globuloviridae: Thermoprotheus tenax spherical virus 1 .

Lipothrixviruses

2005.074B.04-075B.04 To create a new genus in the family Lipothrixviridae and to name it Deltalipothrixvirus.

2005.076B.04 To designate the species Acidianus filamentous virus 2 as the type species of the new genus created in 2005.074B.
2005.077B.04 To create the following as a species of the new genus created in 2005.074B: Acidianus filamentous virus 2 .

Rudiviruses

2005.073B.04 To create the following as a species in the genus Rudivirus in the family Rudiviridae: Acidianus rodshaped virus 1 .

\section{General TPs}

2005.209G.04 To spell out Greek letters in all taxon names, including the names of virus species, in full roman typography: e.g. Alpha, Beta, Gamma, Delta,...Lambda, $M u$, etc. (Note: while this proposal may not be popular in some quarters, it is forced on the ICTV by computer search capabilities which are beyond our control).

\section{ICTV life memberships}

The ICTV Executive Committee unanimously nominates the following eminent virologists and long-term secretaries of the ICTV to Life Membership of the ICTV:

1. Dr. Claude Fauquet

2. Dr. Michael Mayo

Note: Dr. M. Mayo died December 2008.

\section{Conclusion}

As a result of the vote, all of the above taxonomic proposals were approved by the membership. They are now a part of the official ICTV taxonomy. A list of the approved taxa can be found on the ICTV online web site. The most recent report of ICTV was published in 2005 [1]. ICTV is currently updating this information and expects to publish its 9th Report in 2010.

For further information, please visit the ICTV web pages at http://www.ictvonline.org and http://talk.ictvonline.org/.

\section{References}

1. Fauquet C, Mayo MA, Maniloff J, Desselberger U, Ball LA (eds) (2005) Virus taxonomy: eighth report of the International Committee on Taxonomy of Viruses. Elsevier Academic Press, San Diego 